\title{
Publicações de teatro em 2015
}

\author{
LISTA COMPILADA POR SEBASTIANA FADDA
}
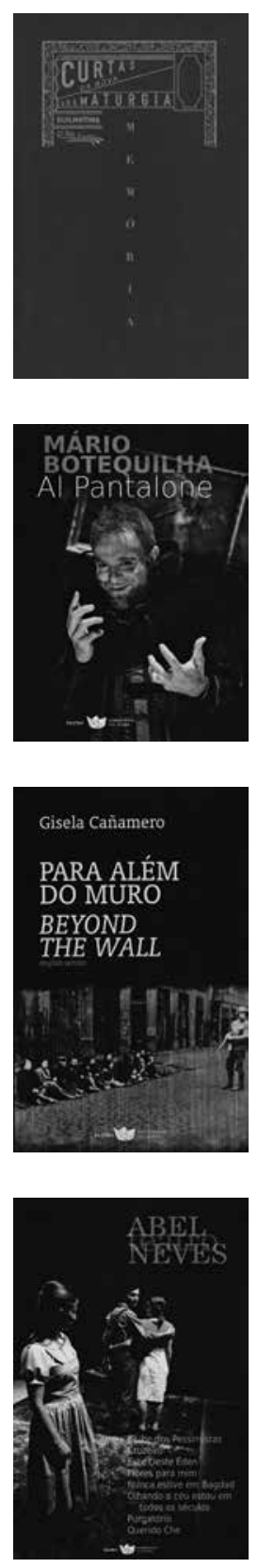

\section{PEÇAS ORIGINAIS (OU VOLUMES DE PEÇAS) EM PRIMEIRA EDIÇÃO}

AA. vV., Curtas da Nova Dramaturgia, n. ${ }^{\circ} 1$ : Memória, Lisboa, Edições Guilhotina, 2015. Botequilha, Mário, Al Pantalone, Lajes do Pico, Companhia das Ilhas, Colecção Azulcobalto Teatro, n. ${ }^{\circ}$ 11, 2015.

CAÑ Amero, Gisela, Para Além do Muro / Beyond the Wall, versão inglesa de Mischa Stocklin, Lajes do Pico, Companhia das Ilhas, Colecção Azulcobalto Teatro, n. ${ }^{\circ}$ 10, 2015.

D. L. R., O Estrangeiro Namorado. Entremez do Século XVIII, ed. José Camões, Bruno Henriques e Ariadne Nunes, Lisboa, Centro de Estudos de Teatro, Faculdade de Letras da Universidade de Lisboa, 2015.

GUEDEs, [Jorge] Castro, Textos de Teatro para a Cena (Estilhaços / Passarox / Gira pró Inferno / Eles Virão, António, Verás Que Virão! / António Vieira, Sementes da Utopia / Os Passos da Paixão de Cristo / Armstrong Viaja de Ptolomeu a Einstein / Lágrima em Fogo / Ecos Verdes / Três Mulheres em Torno de Um Plano / Os Caídos (2084: O Triunfo destes Porcos / Hotel Bilderberg / Entardecer Violeta Rubro / Belém, Ano Zero / Em Defesa da Santa Corporação da Fé Corruptora dos Erros Divinos / Na Capa do Santo: o Natal / Mr. Guantanamero e as Suas Gémeas Queridas / O Cavalo Que Engoliu o Sol. Histórias da Vida de São Paulo / A Sombra do Gafanhoto. Como São João Baptista Deixou de Comer Gafanhotos), Leça da Palmeira, Maçã Vermelha, Associação de Teatro e Cultura, 2015. MARQUes, Raul Malaquias, Ao Vivo e em Direto, Lisboa, Imprensa Nacional-Casa da Moeda, 2015. NETo, Rui, Luto (Monólogo para Um Homem Simples) / Worm (O Estado das Coisas), pref. Paulo Filipe Monteiro, Casal de Cambra, Caleidoscópio, Oro, 2015.

NEves, Abel, Teatro (Clube dos Pessimistas / Cruzeiro / Este Oeste Éden / Flores para Mim / Nunca Estive em Bagdad / Olhando o Céu Estou em Todos os Séculos / Querido Che), Lajes do Pico, Companhia das Ilhas, Colecção Azulcobalto Teatro, n. ${ }^{0}$ 12, 2015.

NEveS-NEVES, Ricardo, Entraria nesta Sala..., Lisboa, Teatro Nacional D. Maria II / Bicho do Mato, 2015 .

Olive IRA, Mickaël de, Obra Completa: 20o6-2014 (Oslo - Fuck Them All and Everything Will Be Wonderful / Boris Yeltsin / 4 Lições para a Sobrevivência / Vou Curar-te pelo Excesso / Hipólito-Monólogo Masculino sobre a Perplexidade), t. I, pref. Fernando Matos Oliveira, com texto de Nuno M. Cardoso e nota do autor, Vila Nova de Famalicão, Húmus, 2015. RODRIgues, Tiago, Ifigénia / Agamémnon / Electra, Lisboa, Teatro Nacional D. Maria II / Bicho do Mato, 2015.

SOARES, Lígia, Romance, Lisboa, Douda Correria, 2015.

TORRADo, António, Maio de 58, Lisboa, Imprensa Nacional-Casa da Moeda, 2015.

Vieir A, Vergílio Alberto, Oratória do Vento / Lenda de Santa Maria Egipcíaca, prólogo Alexei Bueno, desenhos José Monga, s. 1., Crescente Branco, 2015.

WAdDing to n, Gonçalo, Albertine, o Continente Celeste, Lisboa, Abysmo, Colecção Palco, 2015. 

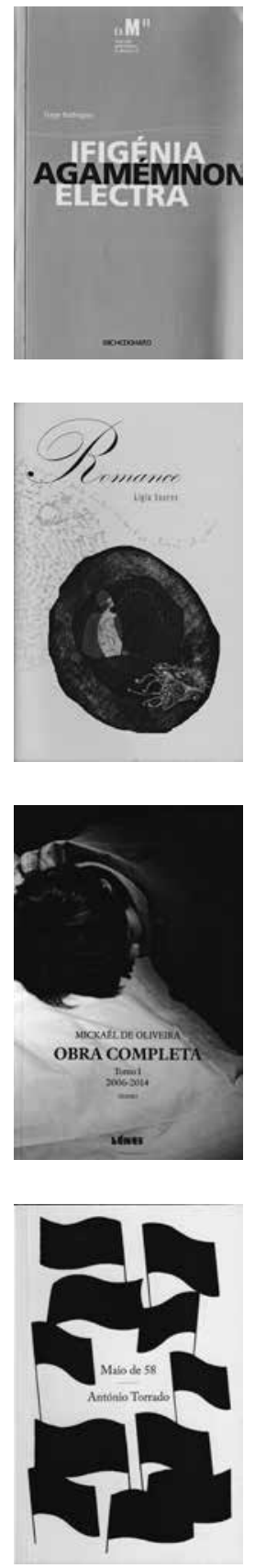

PEÇAS ORIGINAIS (OU VOLUMES DE PEÇAS) EM REEDIÇÃO

TORRADO, António, Salta para o Saco, ilustrações de Carlos Barradas, Lisboa, Editorial Caminho, [2006] 2015, 2. ${ }^{\mathrm{a}} \mathrm{ed}$.

\section{TRADUÇÕES}

ALBEe, Edward, A História do Jardim Zoológico / A Morte de Bessie Smith / Caixa de Areia, trad. Rui Knopfli e Egito Gonçalves, revista por João Vaz, Lisboa, Artistas Unidos / Livros Cotovia, Livrinhos de Teatro n. ${ }^{\circ}$ 89, 2015.

GREIG, David, Os Acontecimentos / Frágil / Dalgety, trad. Pedro Marques, Lisboa, Artistas Unidos / Livros Cotovia, Livrinhos de Teatro n. ${ }^{\circ}$ 86, 2015.

HORvÁTH, Ödön von, Casimiro e Carolina / Histórias do Bosque de Viena, trad. Maria Adélia Silva Melo e Jorge Silva Melo, Lisboa, Artistas Unidos / Livros Cotovia, Livrinhos de Teatro n. ${ }^{\circ}$ 89, 2015 .

JELINEK, Elfriede, Capitalfuck: Os Contratos do Comerciante. Uma Comédia Bancocrática, trad. Helena Topa, textos de Bruno Monteiro, Helena Topa e Emanuel de Sousa, Vila do Conde, Verso da História, 2015.

MÜLLER, Heiner, Filoctetes e Outras Peças (A Batalha / Poemas), trad. José Maria Vieira Mendes, Jorge Silva Melo e João Barrento, Lisboa, Artistas Unidos / Livros Cotovia, Livrinhos de Teatro n. ${ }^{\circ}$ 91, 2015.

--, Quarteto e Outras Peças (Margem Abandonada / Medeia Material / Paisagem com Argonautas / Descrição de Um Quadro), trad. Maria Adélia Silva Melo, Jorge Silva Melo e João Barrento, Lisboa, Artistas Unidos / Livros Cotovia, Livrinhos de Teatro n. ${ }^{\circ}$ 92, 2015 .

PIR AN DEllo, Luigi, O Prazer da Honestidade / Vestir os Nus / A Vida Que Te Dei, trad. Jorge Silva Melo, Lisboa, Artistas Unidos / Livros Cotovia, Livrinhos de Teatro n.o 87, 2015.

PY, Olivier, Epistola aos Jovens Atores para Que Seja Dada a Palavra à Palavra, trad. e apresentação de Maria Luísa Malato, Porto, Deriva, Colecção Pulsar, 2015.

SAR RAZAC, Jean-Pierre, O Fim das Possibilidades, trad. Isabel Lopes, Vila Nova de Famalicão / Porto, Húmus / Teatro Nacional São João, Colecção Teatro Nacional São João, 2015.

SHAKESPEARE, William, Ricardo III, trad., introd. e notas de Rui Carvalho Homem, Lisboa, Relógio D’Água, 2015.

williams, Tennessee, No Bar de Um Hotel de Tóquio e Outras Peças (Ergo-me em Chamas, Gritou a Fénix / A Carta de Amor de Lord Byron / Saudades de Bertha / Casa para Demolição / O Jantar Que não Satisfaz ou A Longa Jornada Interrompida / Fala-me como a Chuva e Deixa-me Ouvir... / Peça para Dois Actores), trad. José Camões e Manuel João Gomes, rev. João Vaz, Lisboa, Artistas Unidos / Livros Cotovia, Livrinhos de Teatro n. ${ }^{\circ}$ 88, Os Clássicos, 2015.

--, Doce Pássaro da Juventude, A Noite do Iguana e Outras Peças (O Zoo de Vidro / Vieux Carré), trad. José Miguel da Silva, José Agostinho Baptista, Dulce Fernandes e Manuel João Gomes, Lisboa, Relógio D’Água, 2015. 

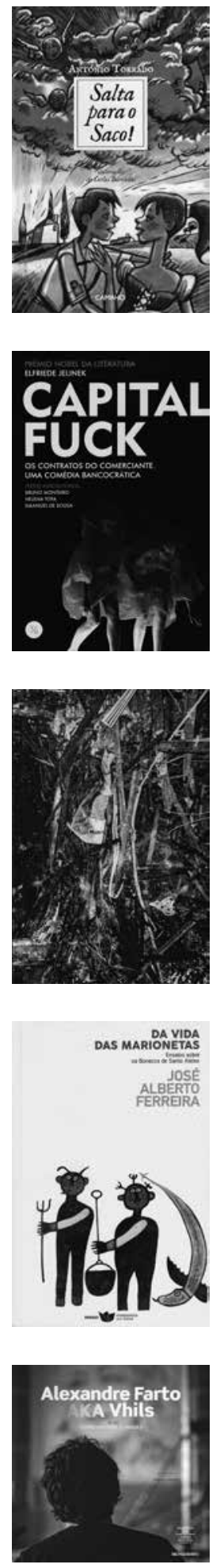

TRADUÇÕES EM REEDIÇÃO

FASSBINDER, Rainer Werner, As Lágrimas Amargas de Petra von Kant, trad. Yvette K. Centeno, Lisboa, Livros Cotovia, Teatro, [1990] 2015, $3 \cdot{ }^{\mathrm{a}}$ ed.

GOETHE, Wolfgang, Fausto, trad. A. F. Castilho, Lisboa, Civilização Editora, Colecção Noites Brancas, [1. ${ }^{\mathrm{a}}$ ed.: Porto, Viúva Moré, 1872] 2015.

SHAKESPEARE, William, Hamlet: Tragédia em 5 actos, ed. bilingue inglês / português, pref. Luís Miguel Cintra, trad. Sophia de Mello Breyner Andresen, revisão com a colaboração do Prof. Grahame Broome-Levett, Lisboa, Assírio \& Alvim, [1. ${ }^{\mathrm{a}}$ ed.: Porto, Lello \& Irmão, 1987] 2015 [pref. de 2015, ligado ao espectáculo levado à cena pelo Teatro da Cornucópia].

--, Hamlet, trad. e notas de António M. Feijó, Lisboa, Relógio D’Água, [1. ${ }^{\mathrm{a}}$ ed.: Lisboa, Livros Cotovia, 2001] 2015.

SófoCles, Rei Édipo, introd., trad. do grego e notas de Maria do Céu Zambujo, Lisboa, Edições 70, Clássicos Gregos \& Latinos, [2008] 2015.

WILDE, Oscar, Um Marido Ideal, trad. Maria Isabel Morna Dias Braga, Porto, Civilização Editora, [1963] 2015.

\section{ESTUDOS / DOCUMENTOS}

BRAnco, António, Visita Guiada ao Ofício do Ator: Um Método, pref. José Trindade Santos, posfácio Manuela de Freitas, Coimbra, Grácio Editor / Centro de Investigação em Artes e Comunicação, 2015.

CATRICA, Paulo, Memorator, Lisboa, Teatro Nacional D. Maria II / Bicho do Mato, Colecção Estudos, 2015.

Cintra, Luís Miguel, Cinco Conversas em Almada, Almada, Companhia de Teatro de Almada, 2015.

Costa, Carlos (2015), Vens Ver ou Vens Viver? Estética e Política da Participação, Lisboa / Paris, Nota de Rodapé Editores, 2015.

CRUz, Hugo (coord.), Arte e Comunidade, Lisboa, Fundação Calouste Gulbenkian, 2015. ESMÉNio, Filipe, O Sonho ao Poder: Mário Viegas. Fotobiografia, Évora, Licorne, 2015.

FARIA, Nuno (coord.), Ricardo Jacinto: O Cone e Outros Lugares, catálogo da exposição organizada pelo Centro Internacional das Artes José de Guimarães, ed. bilingue, trad. inglesa de Martin Dale e Joana Becken, Lisboa, A Oficina / Sistema Solar / Documenta, 2015.

FER REIRA, José Alberto, Da Vida das Marionetas: Ensaio sobre os Bonecos de Santo Aleixo, pref. Christine Zurbach, Lajes do Pico, Companhia das Ilhas / CHAIA (Centro de História de Arte e Investigação Artística) / Secretariado Regional da Cultura / Direcção Regional de Cultura do Alentejo, Colecção Azulcobalto Ensaio n. ${ }^{\circ}$ 2, 2015.

HenriQues, Ricardo / Letria, André, Teatro, Lisboa, Pato Lógico, 2015.

LANCAST RE, Maria José de, Com Um Sonho na Bagagem: Uma Viagem de Pirandello a Portugal, trad. Helena Abreu, Alfragide, Publicações Dom Quixote, 2015.

MCMAHON, Christina, Nações e Transformações em Palco: Festivais de Teatro em Cabo Verde, Moçambique e Brasil, trad. Mick Greer e Graça Margarido em colaboração com Christina S. McMahan e Pedro Lopes de Almeida, Coimbra, Almedina, 2015.

MÜller, Christoph / NEUmAn, Martin (org.), O Teatro em Portugal nos Séculos XVIII e XIX, inclui textos de Christoph Müller, Martin Neumann, Maria Luísa Malato Borralho, Ana Isabel Vasconcelos, Ricarda Musser, Anne Begenat-Neuschäfer e Helmut Siepmann, Actas da secção dedicada ao Teatro em Portugal nos Séculos XVIII e XIX no $8 .^{\circ}$ Congresso Alemão de Lusitanistas [Munique, 2009], Lisboa, Edições Colibri / Ibero-amerikanisches Institut [Berlim], 2015.

PEnA, Abel Nascimento / RELVAs, Maria de Jesus C. / FonseCA, Rui Carlos / CASAL, Teresa (org.), Revisitar o Mito / Myths Revisited, Vila Nova de Famalicão, Húmus, 2015. 

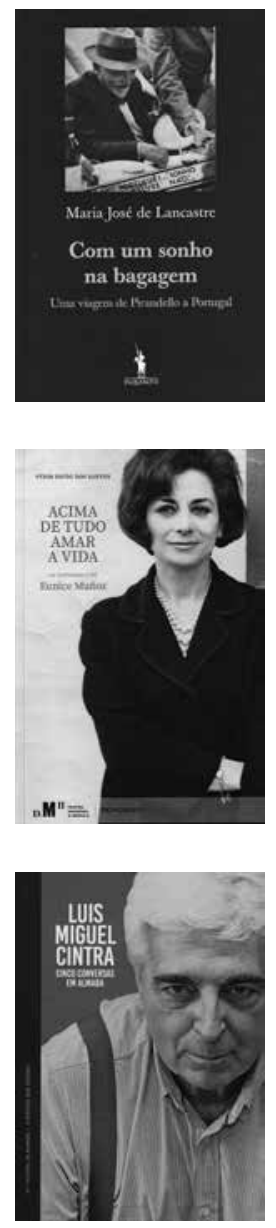

PEREIRA, Silvina, Jorge Pereira de Vasconcelos: Um Homem do Renascimento, catálogo da exposição, rev. técnica Gina Guedes Rafael, rev. A. Miguel Saraiva, Lisboa, Biblioteca Nacional de Portugal, Teatro Maizum, 2015.

SAn Tos, Vítor Pavão, O Veneno do Teatro ou Conversas com Amélia Rey Colaço, Lisboa, Bertrand Editora, 2015.

--, Acima de Tudo Amar a Vida ou Conversas com Eunice Muñoz, Lisboa, Teatro Nacional D. Maria II / Bicho do Mato, 2015.

SILVA, Uirá Iracena, Teatro Popular: Um Conceito Estético-Sociológico, Vila Nova de Famalicão, Húmus, 2015.

VARGAS, Carlos (coord.), Alexandre Farto AKA Vhils no Teatro Nacional D. Maria II, edição bilingue, trad. inglesa de David Bailey, introd. de Miguel Honrado, textos de António Mega Ferreira e Vítor Pavão dos Santos, Lisboa, Teatro Nacional D. Maria II / Bicho do Mato, 2015.

Villa Ret, Henrique, João Villaret (1913-1961): Duas Mãos Que Abertas Deram Tudo, s. 1. [Lisboa], edição de autor, 2015.

\section{ESTUDOS / DOCUMENTOS EM REEDIÇÃO}

VAS QUES, Eugénia, Jorge de Sena: Uma Ideia de Teatro, Lisboa, Guimarães, 2015 [1. a edição: Lisboa, Cosmos, Colecção Literatura, 1998]. 

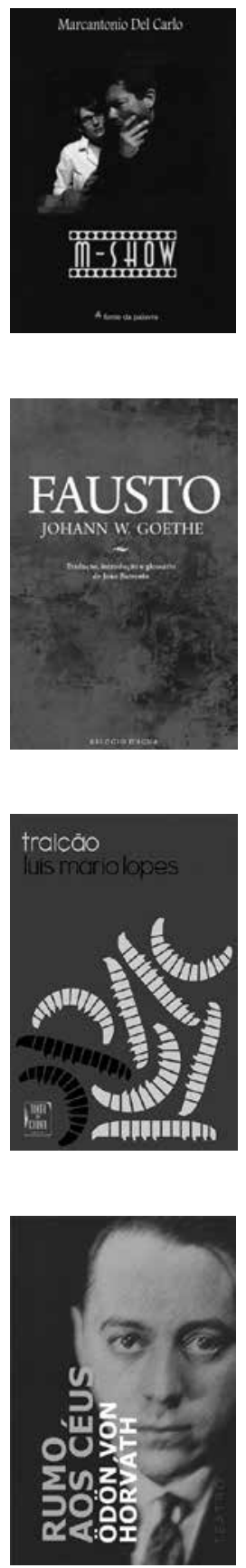

ADENDA À LISTA PUBLICADA NA SINAIS DE CENA N. 21 (2013)

AA. Vv., Kohn: O teatro da corte da Tailândia. Colecção Francisco Capelo, Lisboa, EGEAC / Museu da Marioneta, 2013. [Estudos / documentos]

BECKET T, Samuel, À Espera de Godot, trad. revista e actualizada de José Maria Vieira Mendes, Lisboa, Livros Cotovia, [2006] 2013. [Tradução em reedição actualizada] CORREIA, Hélia, A Teia, Lisboa, Sociedade Portuguesa de Autores / Imprensa Nacional-Casa da Moeda, 2013. [Peça original]

DEL CARLO, Marcantonio, M-Show, Lisboa, Fonte da Palavra, 2013. [Peça original] EURÍPIDES, Medeia, introd., versão do grego e notas de Maria Helena da Rocha Pereira, Lisboa, Fundação Calouste Gulbenkian, [1991, Imprensa de Coimbra] 2013, 5. ${ }^{\mathrm{a}} \mathrm{ed}$. [Tradução em reedição]

GIL, José Manuel Valbom, Teatro Dom Roberto: O teatro tradicional itinerante português de marionetas. O Saloio de Alcobaça e Os Novos Palhetas, ed. ilustrada bilingue, trad. inglesa de Michael Dornan, Lisboa, EGEAC / Museu da Marioneta, 2013. [Estudos / documentos]

GOETHE, Johann W., Fausto, trad., introd. e glossário de João Barrento, Lisboa, Relógio D’Água, [1999, com imagens de Ilda David] 2013. [Tradução em reedição]

HORVÁth, Ödön von, Rumo aos Céus, trad. Maria Gabriela Fragoso, s. l. [Viana do Castelo], Livros de Areia, Colecção Companhia de Teatro de Almada, 2013. [Tradução]

Lopes, Luís Mário, Traição, vencedor do Prémio Luso-Brasileiro de Dramaturgia António José da Silva 2011, Lisboa, Instituto Camões / Tinta-da-china, 2013. [Peça original]

MAMET, David, Negócio Fechado, adaptação de Rodrigo Francisco, s. l. [Viana do Castelo], Livros de Areia, Colecção Companhia de Teatro de Almada, 2013. [Tradução]

PENEDo, Ana Margarida / Lopes, Sofia Campos, Teatro Wayang Kulit de Bali: Objetose coleções do Museu Nacional de Etnologia, Lisboa, Museu Nacional de Etnologia / Imprensa Nacional-Casa da Moeda, 2013. [Estudos / documentos]

Sófocles, Ájax, introd., trad. do grego e notas de Maria Helena da Rocha Pereira, Lisboa, Fundação Calouste Gulbenkian, 2013, 2. a ed. [Tradução em reedição]

\section{ADENDA À LISTA PUBLICADA NA SINAIS DE CENA N. 19 (2012)}

BARros, Né / román, Juan Carlos / maiA, Maria Helena (eds.), Artes Performativas: Novos discursos, Porto, Centro de Estudos Arnaldo Araújo, 2012. [Estudos / documentos]

F RANCo, António Cândido, Autos do Fogo Analógico: Cenas de teatro, Évora, Editora Licorne, 2012. [Peça original]

Lemos, Pedro / vespeira, Marcelino, Adélia e Kovako: Peça de teatro de Pedro Lemose Marcelino Vespeira, Lisboa 195o, edição fac-similada, numerada e assinada, s. l., Sistema Solar, Documenta, 2012. [Peça original em reedição]

SChnit zler, Arthur, Dança de Roda, trad. José A. Palma Caetano, s. l. [Viana do Castelo], Livros de Areia, Colecção Companhia de Teatro de Almada, 2012. [Tradução]

sófocles, Antígona, introd., trad. do grego e notas de Maria Helena da Rocha Pereira, Lisboa, Fundação Calouste Gulbenkian, 2012, 10. ${ }^{\mathrm{a}}$ ed. [Tradução em reedição] TORRADo, António, Atirem-se ao Ar!, Alfragide, Editorial Caminho, 2012. [Peça original]

ADENDA À LISTA PUBLICADA NA SINAIS DE CENA N. 17 (2011)

AA. Vv., Oficina de Escrita Odisseia: Textos escolhidos, Porto, Teatro Nacional São João, 2011. [Peças originais] 

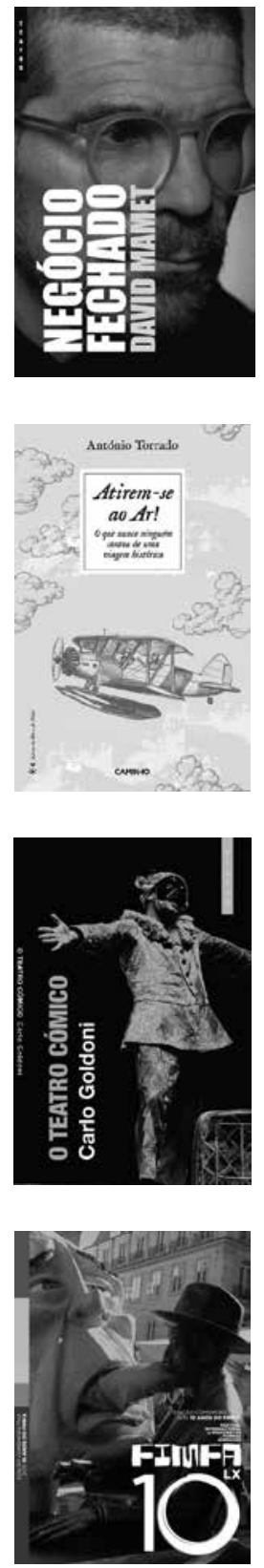

EURÍPIDES, As Bacantes, introd., trad. do grego e notas de Maria Helena da Rocha Pereira, Lisboa, Edições 70, Clássicos Gregos \& Latinos, [1992] 2011. [Tradução em reedição]

Goldoni, Carlo, O Teatro Cómico, trad. José Colaço Barreiros, s. l. [Viana do Castelo], Livros de Areia, Colecção Companhia de Teatro de Almada, 2011. [Tradução]

NORÉ N, Lars, Do Amor, trad. Solveig Nordlund e Bruno Nordlund, s. l. [Viana do Castelo], Livros de Areia, Colecção Companhia de Teatro de Almada, 2011. [Tradução]

ROQUE, Eugénio, Esgrima para Actores: Técnicas universais para criação e representação de duelos em cena, pref. João Vasco, s. l., s. h., 2011. [Estudos / documentos]

SERRES, Karin, Marzïa, trad. Alexandra Moreira de Silva, s. 1. [Viana do Castelo], Livros de Areia, Colecção Companhia de Teatro de Almada, 2011. [Tradução]

\section{ADENDA À LISTA PUBLICADA NA SINAIS DE CENA N. 15 (2010)}

AA. VV., Edição Comemorativa dos 10 Anos do FIMFA Festival Internacional de Marionetase Formas Animadas Lisboa 2010, Lisboa, A Tarumba - Teatro de Marionetas / EGEAC / Museu da Marioneta, 2010. [Estudos / documentos]

Copi, Uma Visita Inoportuna, trad. Jorge Pereirinha Pires, s. 1. [Viana do Castelo], Livros de Areia, Colecção Companhia de Teatro de Almada, 2010. [Tradução]

\section{ADENDA À LISTA PUBLICADA NA SINAIS DE CENA N. 13 (2009)}

AA. Vv., Nadagama: Máscaras e marionetas do Sri Lanka, Lisboa, Museu da Marioneta, 2009. [Estudos / documentos]

AzEvedo, Manuela de, Memória de Uma Mulher de Letras: Poesia, dramaturgia, ficção e ensaio, jornalismo, coord. Etelvina Lage, Ana Maria Tavares e Leonel Gonçalves, pref. Luís Humberto Marcos, Porto, Museu Nacional da Imprensa / Edições Afrontamento, 2009. [Estudos / documentos]

MARIA, Guida / PIN to, Rui Costa, Guida Maria: Uma vida, s. l., ed. Rui Costa Pinto, 2009. [Estudos / documentos]

PINA, Manuel António, História do Sábio Fechado na Biblioteca, ilustrações de Ilda David, Lisboa, Assírio \& Alvim, Colecção Assirinha 24, 2009. [Peça original]

RAmos, Ana Margarida / RoIg Rechou, Blanca-Ana / Gomes, José António (coord.), Teatro para a Infância e Juventude, Porto, Deriva Editores, 2009. [Estudos / documentos]

Simonot, Michel, $O$ Vendedor de Elogios, trad. José Martins, s. 1. [Viana do Castelo], Livros de Areia, Colecção Companhia de Teatro de Almada, 2009. [Tradução]

\section{ADENDA À LISTA PUBLICADA NA SINAIS DE CENA N.11(2008)}

AA. vv., You Can't Go Home Again (ou... A Revolta dos Manequins), catálogo da exposição, Lisboa, EGEAC / Museu da Marioneta, 2008. [Estudos / documentos]

GonzÁlez, Isabel Mociño / Rodriguez, Marta Neira / RAMos, Ana Margarida / silvA, Sara Reis da, Do Livro à Cena, actas dos XIII Encontros Luso-Galaico-Franceses do Livro Infantil e Juvenil, Biblioteca Municipal Almeida Garrett, Porto, 15-17 de Novembro de 2007, Porto, Deriva Editores, 2008. [Estudos / documentos]

TORRADo, António, Verdes São os Campos, lendas teatralizadas do Vale do Minho, Porto, Campo das Letras, [2002] 2008. [Peça original em reedição] 

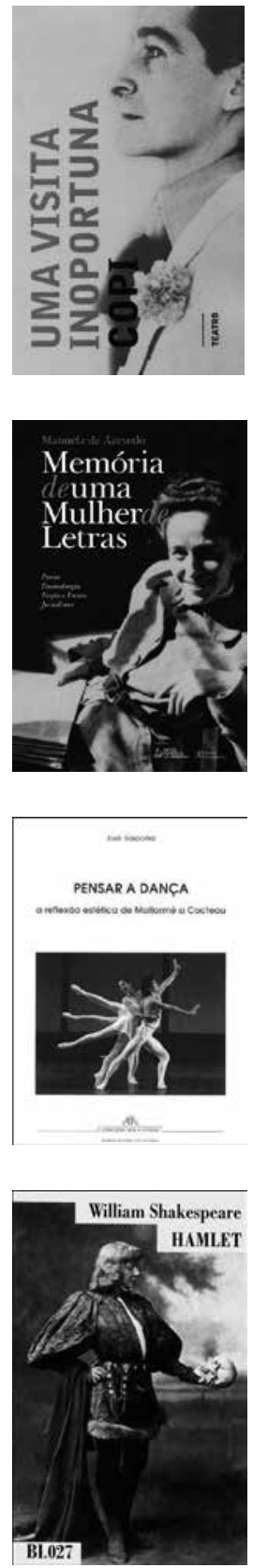

AA. VV., Quando o Corpo Fala. 199o-2005: Imagens de um percurso, pesquisa e coord. Rafael Leitão, s. l. [Évora], Companhia de Dança Contemporânea de Évora, 2007. [Estudos / documentos]

AA. vV., Branca-Flor: O teatro de Lília da Fonseca 1962-1982, catálogo da exposição, Lisboa, EGEAC / Museu da Marioneta, 2007. [Estudos / documentos]

ALMEIDA, Carmen (coord.), Mestre Salas Apresenta: Exposição de marionetas portuguesas, Évora, Direcção Regional da Cultura do Alentejo em colaboração com a Câmara Municipal de Évora e o CENDREV, 2007. [Estudos / documentos]

ARTAud, Antonin, Eu, Antonin Artaud, trad. e apresentação de Aníbal Fernandes, Lisboa, Assírio \& Alvim, 2007. [Estudos / documentos]

FRANCISCo, Rodrigo, Quarto Minguante, s. l. [Viana do Castelo], Livros de Areia, Colecção Companhia de Teatro de Almada, 2007. [Peça original]

KEene, Daniel, Pedra, Papel e Tesoura, trad. Maria Arriaga, s. 1. [Viana do Castelo], Livros de Areia, Colecção Companhia de Teatro de Almada, 2007. [Tradução]

Shakespeare, William, Hamlet, trad. António M. Feijó, Rio do Mouro, Biblioteca de Editores Independentes, BI 027, [1. a ed.: Lisboa, Livros Cotovia, 2001] 2007. [Tradução em reedição]

ADENDA À LISTA PUBLICADA NA SINAIS DE CENA N. 7 (2006)

SASPORTEs, José, Pensar a Dança: A reflexão estética de Mallarmé a Cocteau, introd. Fedele D’Amico, Lisboa, Imprensa Nacional-Casa da Moeda, Colecção Arte e Artistas, 2006, 2. ${ }^{\mathrm{a}}$ ed. revista e aumentada. [Estudos / documentos]

SHAKESPEARE, William, Hamlet, trad. António M. Feijó, Lisboa, Livros Cotovia, 2006. [Tradução]

\section{ADENDA À LISTA PUBLICADA NA SINAIS DE CENA N. 5 (2005)}

MElo, Maria do Céu de, A Expressão Dramática à Procura de Percursos, Lisboa, Livros Horizonte, 2005. [Estudos / documentos - Errata: Rectificação do título]

TORRADo, António, Tudo Corre Bem no Melhor dos Mundos, tragicomédia voltairiana, Lisboa, Página 4, Colecção Antestreia, 2005. [Peça original]

\section{ADENDA À LISTA PUBLICADA NA SINAIS DE CENA N. $1(2003)$}

TOR RADo, António, Lugar Sagrado, peça em 1 acto, Lisboa, Página 4, Colecção Antestreia, 2003. [Peça original] 